\title{
An Efficient Integrated Approach for the Detection of Exudates and Diabetic Maculopathy in Colour fundus Images
}

\author{
B.Ramasubramanian ${ }^{1}$ and G.Mahendran ${ }^{2}$ \\ ${ }^{1}$ Department of Electronics and Communication Engineering, \\ Syed Ammal Engineering College, Ramanathapuram, Tamil Nadu, India. \\ ramatech87@gmail.com \\ ${ }^{2}$ Department of Electronics and Communication Engineering, \\ Syed Ammal Engineering College, Ramanathapuram, Tamil Nadu, India.
}

\begin{abstract}
Diabetic Retinopathy $(D R)$ is a major cause of blindness. Exudates are one of the primary signs of diabetic retinopathy which is a main cause of blindness that could be prevented with an early screening process In this approach, the process and knowledge of digital image processing to diagnose exudates from images of retina is applied. An automated method to detect and localize the presence of exudates and Maculopathy from low-contrast digital images of Retinopathy patient's with non-dilated pupils is proposed. First, the image is segmented using colour K-means Clustering algorithm. The segmented image along with Optic Disc (OD) is chosen. To Classify these segmented region, features based on colour and texture are extracted. The selected feature vector are then classified into exudates and nonexudates using a Support Vector Machine (SVM) Classifier. Also the detection of Diabetic Maculopathy, which is the severe stage of Diabetic Retinopathy is performed using Morphological Operation. Using a clinical reference standard, images with exudates were detected with $96 \%$ success rate. This method appears promising as it can detect the very small areas of exudates.
\end{abstract}

\section{Keywords}

CIE Lab Colour Space, CLAHE, Diabetic Retinopathy (DR), Exudates, GLCM, K-Means Clustering, SVM.

\section{INTRODUCTION}

Diabetic Retinopathy is the common retinal complication associated with diabetes. It is a major cause of blindness in both middle and advanced age groups [1]. The International Diabetes Federation reports that over 50 million people in India have this disease and it is growing rapidly (IDF 2009a) [2]. The estimated prevalence of diabetes for all age groups worldwide was $2.8 \%$ in 2000 and $4.4 \%$ in 2030 meaning that the total number of diabetes patients is forecasted to rise from 171 million in 2000 to 366 million in 2030 [3]. Therefore regular screening is the most efficient way of reducing the vision loss.

Diabetic Retinopathy is mainly caused by the changes in the blood vessels of the retina due to increased blood glucose level. Exudates are one of the primary sign of Diabetic Retinopathy [5]. Exudates are yellow-white lesions with relatively distinct margins. Exudates are lipids and proteins that deposits and leaks from the damaged blood vessels within the retina. Detection of Exudates by ophthalmologists is a laborious process as they have to spend a great deal of time in manual analysis and diagnosis. Moreover, manual detection requires using chemical dilation material which takes time and has negative side effects on patients. Hence automatic screening techniques for exudates detection have great significance in saving costs, time and labour in addition to avoiding the side effects on patients.

DOI : 10.5121/acij.2012.3509 
Digital Colour fundus images are widely used by ophthalmologists for diagnosing Diabetic Retinopathy. DR also causes numerous abnormalities like microaneurysm, haemorrhages, cotton wool spots, neo-vascularisation and in later stages, retinal detachment.

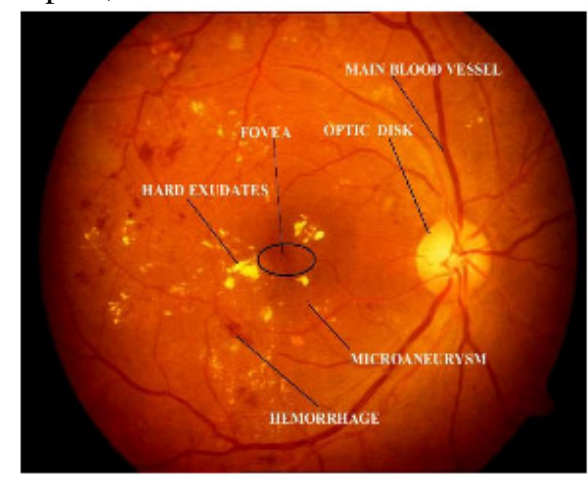

Figure 1. Diabetic Retinopathy image with various typical components.

Figure 1 depicts a typical retinal image labelled with various feature components of Diabetic Retinopathy. Microaneurysm are small saccular pouches and appears as small red dots. This may lead to big blood clots called haemorrhages. The bright circular region from where the blood vessels emanate is called optic disk (OD). Macula is the centre portion of the retina and has photoreceptors called cons that are highly sensitive to colour and responsible for perceiving fine details. It is situated at the posterior pole temporal to the optic disk. The fovea defines the centre of the macula and is the region of highest visual acuity.

\section{PROPOSED METHOD FOR THE DETECTION OF EXUDATES IN COLOUR FUNDUS IMAGES:}

\subsection{State of Art:}

Alireza Osareh et al [4] proposed a method for automatic identification of exudates based on computational Intelligence technique The colour retinal images were segmented using fuzzy cmeans clustering. Feature vector were extracted and classified using multilayer neural network classifier.

Akara Sopharak et al [5] reported the result of an automated detection of exudates from low contrast digital images of retinopathy patients with non-dilated pupils by Fuzzy C-Means clustering. Four features such as intensity, standard deviation on intensity, hue and a number of edge pixels were extracted and applied as input to coarse segmentation using FCM clustering method. The detected result were validated with expert ophthalmologists hand drawn ground truths. Sensitivity, Specificity, positive predictive value (PPV), positive likelihood ratio (PLR) and accuracy were used to evaluate the overall performance of the system.

Niemeijer et al [6] distinguished the bright lesion like exudates, cotton wool spots and drusen from colour retinal images. In the first step, pixels were classified, resulting in a probability map that included the probability of each pixel to be part of a bright lesion. Then, pixels with high probability were grouped into probable lesion pixel clusters. Based on cluster characteristics, each cluster was assigned a probability indicating the likelihood that the cluster was a true bright lesion. Finally these clusters were classified as exudates, cotton wool spots or drusen. Sensitivities and specificities of the annotations on the 300 images by the automated system were obtained.

Akara sopharak et al [7] proposed a series of experiments on feature selection and exudates classification using naive bayes and Support Vector Machine (SVM) Classifiers. At first, they used naive bayes model to a training set consisting of 15 features extracted from 
positive and negative examples of exudates pixels. Next, to obtain the best SVM, they used the best feature set from the naive bayes classifier and continually appended the removed features to the classifier. For each combination of features, they carried out a grid search to find the best combination of hyper parameters like tolerance for training error and radial basis function width. They compared the best naive bayes and SVM classifier to a Nearest Neighbour classifier. They proved that the naive bayes and SVM classifiers executed better than the NN classifier.

Walter et al [8] identified exudates from green channel of the retinal images according to their gray level variation. The exudates contour were determined using mathematical morphology techniques. This method used three parameters: size of the local window and two threshold value. Exudates regions were initially found using first threshold value. The second threshold represents the minimum value, by which a candidate pixel must differ from its surrounding background to be classified as exudates. The author achieved a sensitivity of $92.8 \%$ and predictivity of $92.4 \%$ against a set of 15 abnormal retinal images. However the author ignored some types of errors on the border of the segmented exudates in their reported performances and did not discriminate exudates from cotton wool spots.

Sinthanayothin et al [9] reported the result of an automated detection of Diabetic Retinopathy by Recursive Region Growing techniques on a 10X10 window using selected threshold values. In the pre-processing steps, adaptive, local, contrast enhancement is applied. The author reported a sensitivity of $88.5 \%$ and specificity of $99.7 \%$ for the detection of exudates against a small dataset comprising 21 abnormal and 9 normal retinal images.

Phillips et al [10] identified the exudates by using Global and local thresholding. The input images were pre-processed to eliminate photographic non-uniformities and the contrast of the exudates was then enhanced. The lesion based sensitivity of this technique was reported between $61 \%$ and $100 \%$ based on 14 images. A drawback of this method was that other bright lesions (such as cotton wool spots) could be identified mistakenly.

\subsection{Image Acquisition:}

To evaluate the performance of this method, the digital retinal images were acquired using Topcon TRC-50 EX camera with a 50 ${ }^{\circ}$ field of view at Aravind Eye hospital, Madurai.

\subsection{Pre-processing:}

Colour fundus images often show important lighting variation, poor contrast and noise. In order to reduce these imperfection [11] and generate images more suitable for extracting the pixel features in the classification process, a pre-processing comprising the following step is applied. 1) RGB to HSI conversion 2) Median Filtering 3) Contrast Limited Adaptive Histogram Equalization (CLAHE).

\subsubsection{RGB to HSI Conversion:}

The input retinal images in RGB Colour space are converted to HSI colour space. The noise in the images are due to the uneven distribution of the intensity(I) component.

\subsubsection{Median Filtering:}

In order to uniformly distribute the intensity throughout the image, the I-component of HSI colour space is extracted and filtered out through a $3 \mathrm{X} 3$ median filter.

\subsubsection{Contrast Limited Adaptive Histogram Equalization (CLAHE):}

The contrast limited adaptive histogram equalization is applied on the filtered I-component of the image [12]. The histogram equalized I component is combined with HS component and transformed back to the original RGB colour space. 


\subsection{Image Segmentation based on K-Means:}

In this approach, we present a novel image segmentation based on colour features from the images. The work is divided into two stages: First, enhancing the colour separation is done by extracting the $a^{*} b^{*}$ components from the $L^{*} a^{*} b^{*}$ colour space of the pre-processed image. Then, the regions are grouped into a set of five clusters using K-means Clustering algorithm. By this two step process, we reduce the computational cost avoiding feature calculation for every pixel in the image[13].

The entire process can be summarized in following steps:

Step 1: Read the image. Figure 2 shows the example input retinal image with exudates.

Step 2: Convert the image from RGB colour space to $L^{*} a * b *$ colour space (Figure 3). L*a*b* colour space helps us to classify the colour differences. It is derived from the CIE XYZ tristimulus values. $\mathrm{L}^{*} \mathrm{a}^{*} \mathrm{~b}^{*}$ colour space consists of a Luminosity layer $\mathrm{L}^{*}$, chromaticity layer $a^{*}$ indicating where the colour falls along the red-green axis, chromaticity layer $b^{*}$ indicating where the colour falls along the blue-yellow axis. All of the colour information is in the $\mathrm{a}^{*}$ and $b^{*}$ layer. The difference between two colours can be measured using the Euclidean distance.

Step 3: Segment the colours in $\mathrm{a}^{*} \mathrm{~b}^{*}$ space using K-means clustering. Clustering is a way to separate groups of objects. K-Means Clustering treats each object as having a location in space. It finds partition such that objects within each cluster are close to each other as possible and as far from other objects in other clusters as possible. The algorithm requires that we specify the number of clusters to be partitioned and a distance metric to quantify how close two objects are to each others. Since the colour information exist in the $a^{*} b^{*}$ space, our objects are pixels with $a^{*}$ and $b^{*}$ values. Use K-means to cluster the objects into five clusters using the Euclidean distance metric.

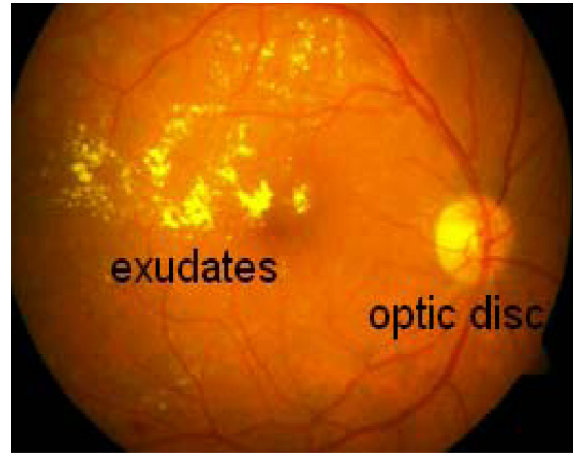

Figure 2. Original Image

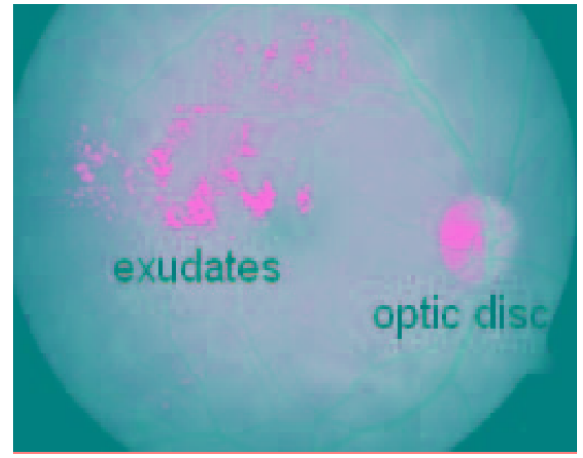

Figure 3. CIE Lab Colour space

conversion.

Step 4: Label every pixel in the image using the result from K-means For every objects in the input, K-means returns an index corresponding to a cluster. Label every pixel in the image with its cluster index.

Step 5: Create images that segment the images by colour.

Step 6: Since the Optic Disc and Exudates are homogenous in their colour property, cluster possessing Optic Disc is localized for further processing.

\subsection{Feature Extraction:}

To classify the localized segmented image into exudates and Non-exudates, a number of features based on colour and texture are extracted using Gray Level Co-occurrence Matrix (GLCM) . GLCM is a tabulation of how often different combination of pixel brightness values occur in a pixel pair in an image. Each element $(i, j)$ in GLCM specifies the number of times 
that the pixel with value $\mathrm{i}$ occurred horizontally adjacent to a pixel with value $\mathrm{j}$. The resulting matrix was analysed and based on the existing information, the feature vectors are formed [14].

Based on texture, the following features are extracted:

Table 1. Feature Extraction

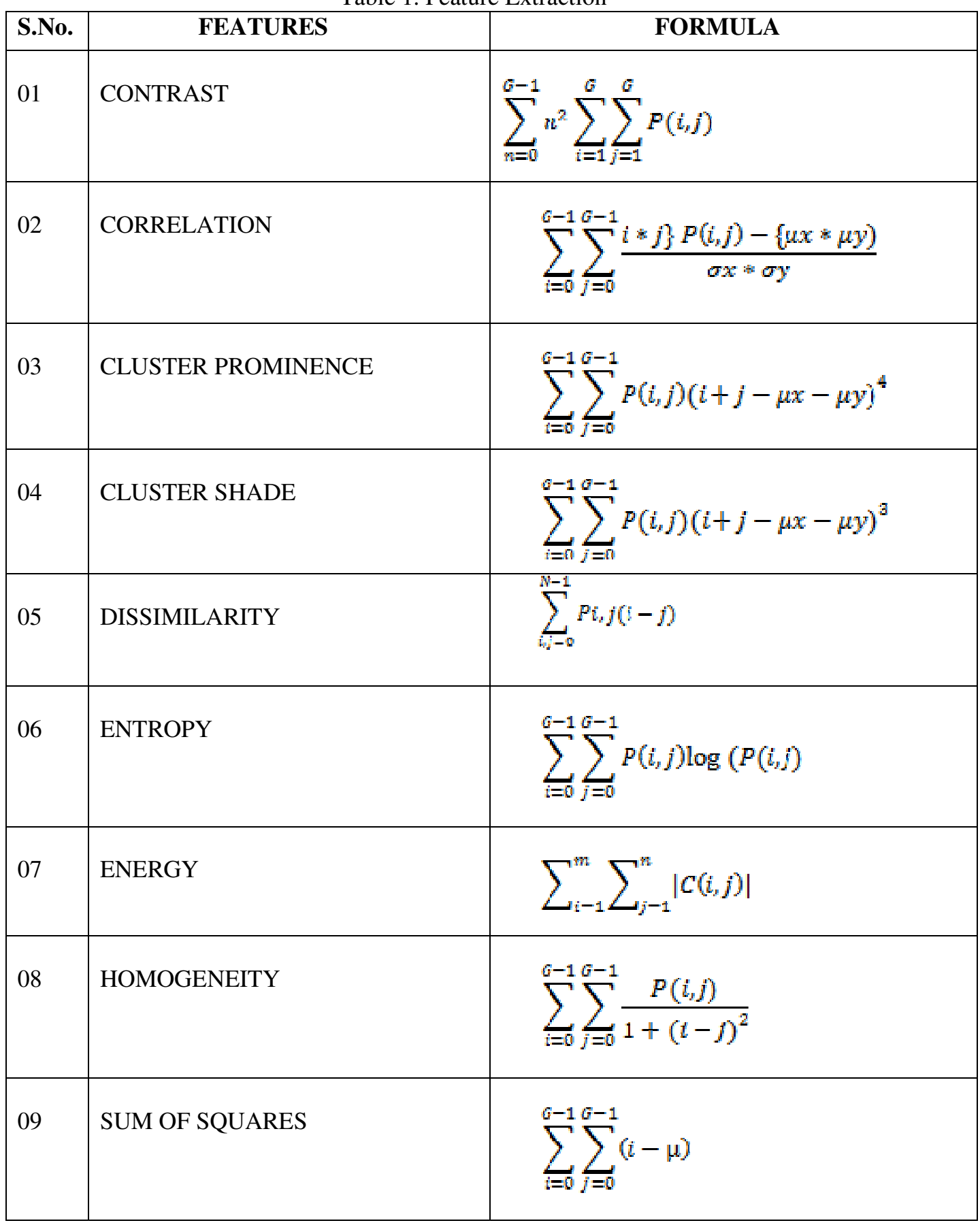




\subsection{Classification using SVM:}

To classify these segmented region into Exudates and Non-Exudates, we make use of wellknown efficient Support Vector Machine (SVM) Classifier. The System is trained using the above calculated feature vectors and the method is tested on 70 abnormal images and 30 normal images. SVM classifier outperforms well when compared with other types of classifiers.

\subsection{Detection of Diabetic Maculopathy :}

For the Detection of Diabetic Maculopathy, the Green Component is extracted from the colour fundus images. It is then pre-processed using the median filter and Adaptive Histogram Equalization. The Green component is applied to Bottom-hat transform and the result is subtracted with the top-hat transformed image. By applying these transform, macula, which is the darkest region of an image is detected. If the exudates is present in the macula, then it indicates the presence of Diabetic Maculopathy.

\subsection{Result:}

\subsubsection{Exudates Detection using SVM:}

In this approach, we have investigated and proposed a method to automatically extract exudates from Diabetic Retinopathy images. The pre-processed colour retinal image is segmented into five cluster using colour K-Means Clustering algorithm. The cluster containing Optic Disc is selected and features are extracted. The segmented colour image with Optic Disc is shown in Figure 4.b.

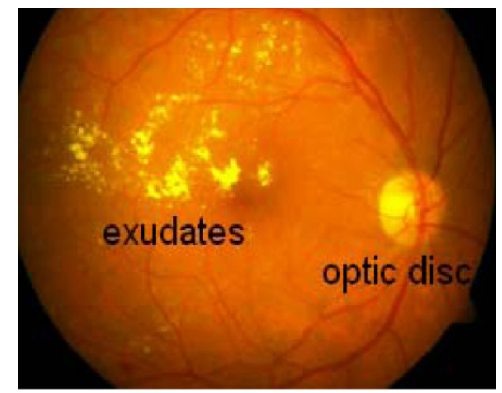

Figure 4.a. Original Image

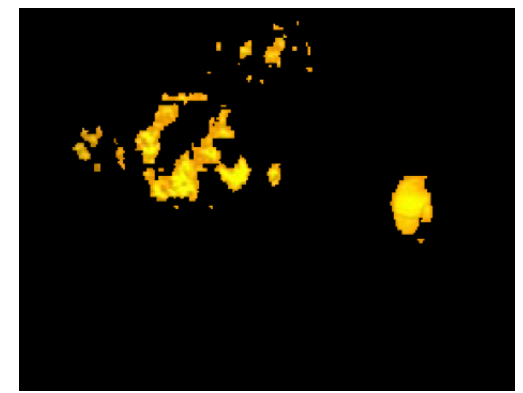

b. Selected Cluster with Optic Disc.

In the selected cluster, feature vector are extracted using Gray Level Co-occurrence Matrix. With the help of these features, the selected cluster is classified into Normal (Non- Exudates) or Abnormal (Exudates) using Support Vector Machine (SVM) Classifier. The success rate is found to be $96 \%$.

Figure 5 shows the entire GUI based system for the detection of Exudates in Diabetic Retinopathy Images. 


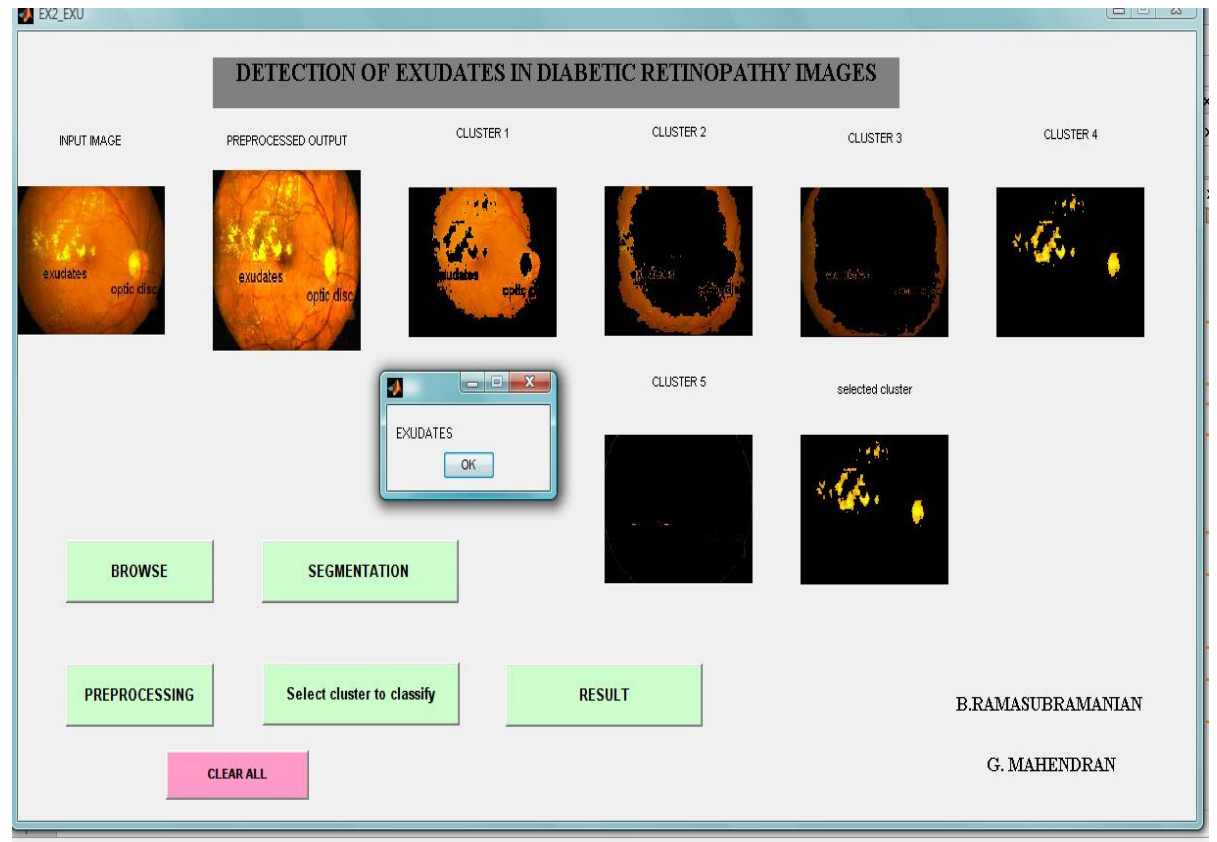

Figure 5. Detection of Exudates in Diabetic Retinopathy Images.

\subsubsection{Detection of Diabetic Maculopathy:}

Macula is the darkest part in the retinal image. This region is localized using morphological operation. If exudates is present in this macula region, then it indicates the presence of Diabetic Maculopathy. If exudates is not present in this region, then it shows the absence of Diabetic Maculopathy.

Figure 6.a shows the colour fundus image affected with Diabetic Maculopathy. The centre darkest region is the macula. In our approach, the Diabetic Maculopathy is detected using morphological operation and it is indicated in Figure 6.b using the rectangular box.

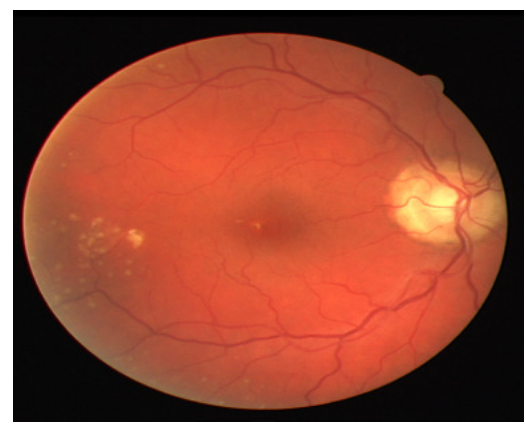

Figure 6. a) Colour fundus Image

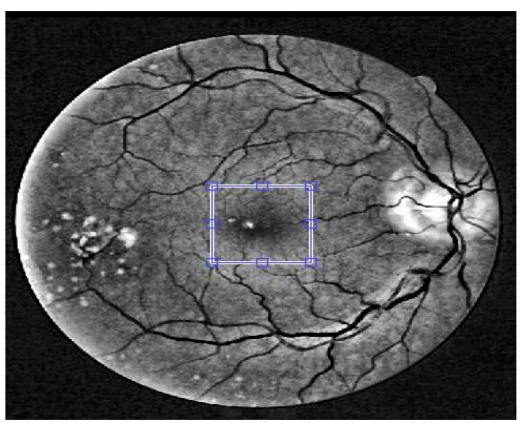

b) Detection of Diabetic Maculopathy

\section{CONCLUSIONS:}

The diabetic retinopathy images were collected from STARE and DRIVE database. Exudates are one of the earlier signs of diabetic retinopathy. The low contrast digital image is enhanced using Contrast Limited Adaptive Histogram Equalization (CLAHE). The noise are removed from the images using median filter. The Contrast enhanced colour image is segmented using 
K-means clustering, which is one of the simplest unsupervised learning algorithm for image segmentation. algorithm. K-means clustering takes less computational time compared to FCM. It provides more colour information from which the result of classification will be improved. To Classify these segmented image into exudates and non-Exudates, a set of features based on texture and colour are extracted using Gray Level Co-Occurrence Matrix (GLCM). The selected features are classified into exudates and non-exudates using Support Vector Machine (SVM) Classifiers. Also the detection of Diabetic Maculopathy, which is the severe stage of Diabetic Retinopathy is performed using morphological operation. The method is evaluated on 70 abnormal and 30 normal images. Out of these 100 images, 96 images were detected successfully and thus a success rate of $96 \%$ was obtained and Diabetic Maculopathy are detected with $100 \%$ success rate.

\section{ACKNOWLEDGEMENTS}

Our Sincere thanks to Department of Ophthalmology, Vasan Eye Care Hospital, Chennai, TamilNadu for providing the images and necessary details.

\section{REFERENCES}

[1] Olson. J. A, Strachana. F.M, Hipwell. J. H, “A comparative evaluation of digital imaging, retinal photography and optometrist examination in screening for diabetic retinopathy" Journal on Diabet Med. Vol. 20, No. 7 .pp. 528- 534, July 2003.

[2] International Diabetic Federation (IDF), 2009a, Latest diabetes figures paint grim global picture.

[3] Saiprasad Ravishankar, Arpit Jain, Anurag Mittal, "Automated feature extraction for early detection of Diabetic Retinopathy in fundus images". IEEE Conference on Computer vision and pattern Recognition, pp. 210-217, August 2009.

[4] Alireza Osareh, Bita shadgar and Richard Markham,"A computational intelligence based approach for detection of exudates in Diabetic Retinopathy Images", IEEE Trans. on Information Technology in Biomedicines, vol. 13, no. 4, pp. 535-545, July 2009.

[5] Akara Sopharak, Bunyarit Uyyanonvara, Sarah Barman, "Automatic Exudate Detection from Non-dilated Diabetic Retinopathy retinal images using Fuzzy C-Means Clustering” Journal of Sensors, vol.9, No. 3, pp 2148- 2161, March 2009.

[6] Niemeijer. B.V, Ginnekan. S. R, Russell. M, and M. D. Abramoff, “Automated detection and differentiation of drusen, exudates and cotton- wool spots in digital color fundus photographs for diabetic retinopathy diagnosis", Journal on Investigate Ophthalmol. And Visual Science., vol. 48, No. 2 pp. 2260-2267, 2007.

[7] Akara Sopharak, Mathew N. Dailey, Bunyarit Uyyanonvara, Sarah Barman, Tom Williamson, Yin Aye Moe, "Machine Learning approach to automatic Exudates detection in retinal images from diabetic patients", Journal of Modern optics, Vol. 57, No. 2, pp. 124-135, Nov 2011.

[8] T. Walter, J.Klein, P.Massin and A.Erginary, "A Contribution of image processing to the diagnosis of Diabetic Retinopathy detection of exudates in color fundus images of the human retina”, IEEE Trans. On Med. images, vol. 21, no. 10, pp. 1236-1243, 2002.

[9] C. Sinthanayothin, "Image analysis for automatic diagnosis of Diabetic Retinopathy", Journal of Medical Science, Vol. 35,No. 5, pp. 1491-1501, Jan 2011.

[10] Fleming. AD, Philips. S, Goatman. KA, Williams. GJ, Olson. JA, sharp. PF, “Automated detection of exudates for Diabetic Retinopathy Screening”, Journal of Phys. Med. Bio., vol. 52, no. 24, pp. 7385-7396, 2007.

[11] Guoliang Fang, Nan Yang, Huchuan Lu and Kaisong Li, "Automatic Segmentation of Hard Exudates in fundus images based on Boosted Soft Segmentation", International Conference on Intelligent Control and Information Processing, pp. 633-638, Sept 2010. 
Advanced Computing: An International Journal ( ACIJ ), Vol.3, No.5, September 2012

[12] Pizer. S.M. "The Medical Image Display and analysis group at the university of North Carolina:Reminiscences and philosophy” IEEE Trans On Medical Imaging, vol. 22, no. 1, pp. 2-10, April 2003.

[13] Plissiti.M.E., Nikar.C, Charchanti.A, "Automated detection of cell nuclei in pap smear images using morphological reconstruction and clustering" IEEE Trans. On Information Technology in Biomedicine, vol.15,no. 2, pp. 233-241, March 2011.

.[14] Seongijin park, Bohyoung Kim, Jeongjin Loe“ GGO nodule volume preserving Non-rigid Lung Registration using GLCM texture analysis”, IEEE Trans. On Biomedical Engg., vol. 58, no. 10, pp. 2885-2894, sept 2011.

[15] Kandaswamy.U, Adjerch.D.A, Lee.M.C, "Efficient Texture analysis of SAR imagery”, IEEE

Trans. On Geoscience and Remote Sensing, vol. 43, no. 9,pp. 2075-2083, August 2005.

[16] Tobin.K.N, Chaum.E, Govindasamy.V.P, "Detection of anatomic structures in human retinal imagery"

IEEE Transactions on medical imaging, vol. 26, no. 12,pp. 1729-1739, December 2007.

[17] Gwenole Quellec, Stephen R. Russell, and Michael D. Abramoff, Senior Member, IEEE "Optimal Filter Framework for Automated, Instantaneous Detection of Lesions in Retinal Images" IEEE Trans. on medical imaging, vol. 30, no. 2,pp. 523-533, February 2011.

[18] Akara Sopharak, Bunyarit Uyyanonvara, sarah Barman, "Comparative analysis of automatic exudates detection algorithms", Proceedings of the world congress on Engg., Vol I, Dec 2011.

[19] Doaa Youssef, Nahed Solouma, Amr El-dib, Mai Mabrouk, "New Feature-Based Detection of Blood Vessels and Exudates in Color Fundus Images" IEEE conference on Image Processing Theory, Tools and Applications,2010,vol.16,pp.294-299.

\section{Author}

B.Ramasubramanian received his B.E Degree from Syed Ammal Engineering College, Ramanathapuram, TamilNadu, India in 2008 and M. Tech Degree from B. S. Abdur Rahman University, Chennai, TamilNadu, India in 2012. He is currently an Assistant Professor in Syed Ammal Engineering College, Ramanathapuram. His Research area includes medical image processing, Digital Signal Processing and Machine Learning.

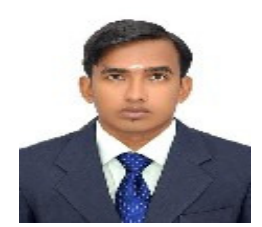

G. Mahendran received his B.E Degree from Mohamed Sathak Engineering College, Ramanathapuram, TamilNadu, India and M. E Degree from Mohamed Sathak Engineering College, TamilNadu, India. He is currently an Assistant Professor in Syed Ammal Engineering College, Ramanathapuram. His Research area includes Digital Image processing and Digital Signal Processing.. 